\title{
Mechanism of Rare Earth Incorporation and Crystal Growth of Rare Earth Containing Type-I clathrates
}

Andrey Prokofiev $^{1 *}$, Robert Svagera $^{1}$, Monika Waas ${ }^{1}$, Matthias Weil ${ }^{2}$, Johannes Bernardi ${ }^{3}$ and Silke Paschen $^{1}$

${ }^{1}$ Institute of Solid State Physics, Vienna University of Technology, Wiedner Hauptstr. 8-10, Vienna 1040 Austria ${ }^{2}$ Institute of Chemical Technologies and Analytics, Vienna University of Technology, Getreidemarkt 9/164-SC, 1060 Vienna, Austria

${ }^{3}$ USTEM, Wiedner Hauptstr. 8-10, Vienna 1040 Austria 


\section{X-ray powder diffraction of the $\mathrm{Ba}_{6} \mathrm{Ce}_{2} \mathrm{TM}_{6} \mathrm{Si}_{40}(\mathrm{TM}=\mathrm{Au}, \mathrm{Pt}, \mathrm{Pd})$ samples}

$\mathrm{TM}=\mathrm{Au}$
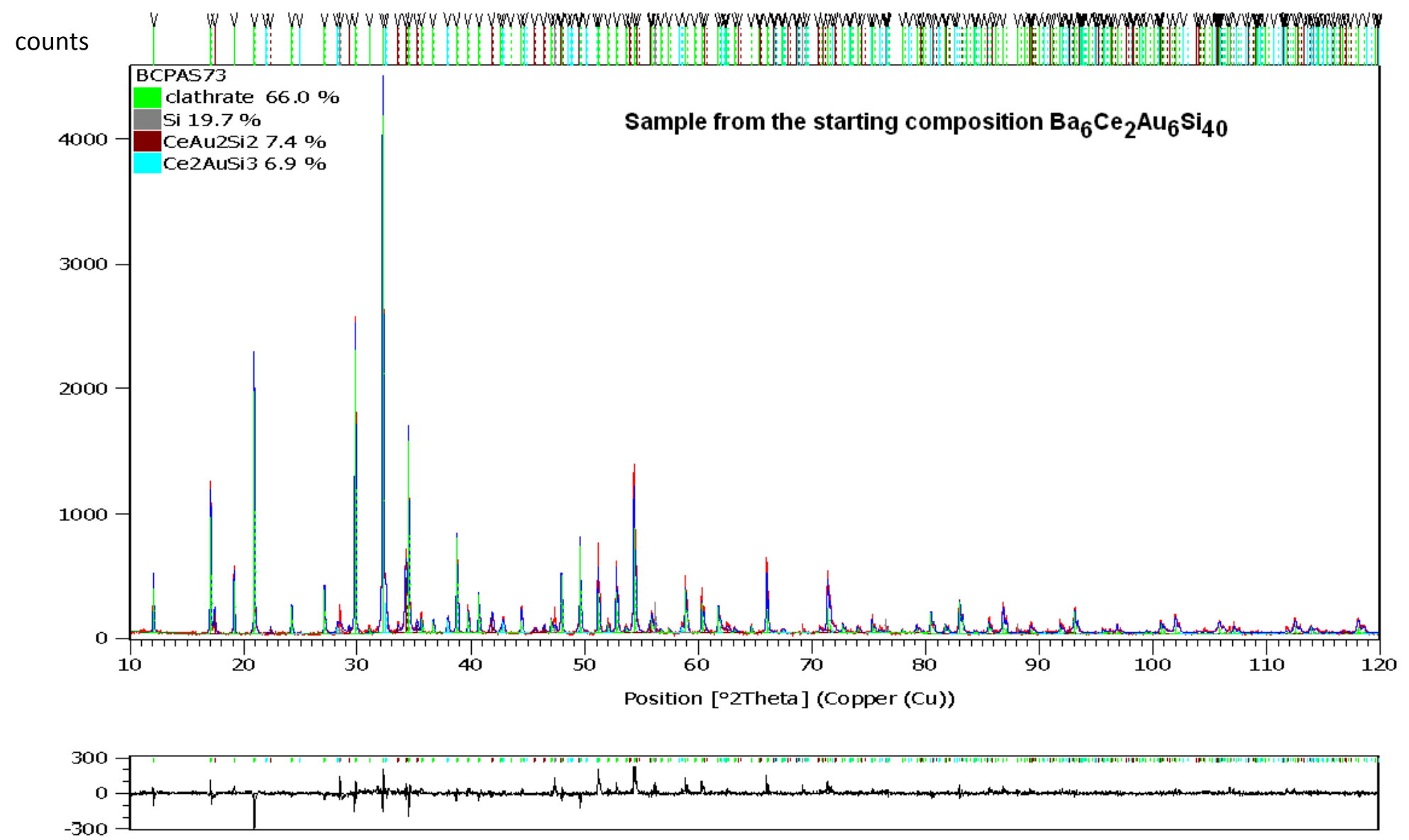

\section{$\mathrm{Ba}_{6} \mathrm{Ce}_{2} \mathrm{Au}_{6} \mathrm{Si}_{40}$}

Clathrate phase $(\mathrm{Ba}, \mathrm{Ce})_{8.00} \mathrm{Au}_{5.40} \mathrm{Si}_{40.60}$

$a=10.3989(2) \AA$

Secondary Phases:

1. $\mathrm{Si}$,

2. (Ce, Ba)Au $\mathrm{Ai}_{2}$ (S.G. $14 / \mathrm{mmm}$ ). Structure type $\alpha-\mathrm{ThCr}_{2} \mathrm{Si}_{2}$ (Ref. 19)

$a=4.3223(2) \AA$

c=10.1938(4) ^,

3. $\mathrm{Ce}_{2} \mathrm{AuSi}_{3}$ (S.G. 14 /amd). Structure type $\alpha-\mathrm{ThSi}_{2}$ (Ref. 20)

$\mathrm{a}=4.2262(2) \AA$

$c=14.3191(4) \AA$ 
$\mathrm{TM}=\mathbf{P t}$

\section{$\mathrm{Ba}_{6} \mathrm{Ce}_{2} \mathrm{Pt}_{6} \mathrm{Si}_{40}$}

Clathrate phase $(\mathrm{Ba}, \mathrm{Ce})_{8.00} \mathrm{Pt}_{3.60} \mathrm{Si}_{42.40}$

$a=10.3511(2) \AA$

Secondary Phases:

counts BCPAS93 clathrate $52.6 \%$ Si $6.1 \%$

CePtSi3 $41.3 \%$

Sample from the starting composition $\mathrm{Ba}_{6} \mathrm{Ce}_{2} \mathrm{Pt}_{6} \mathrm{Si}_{40}$
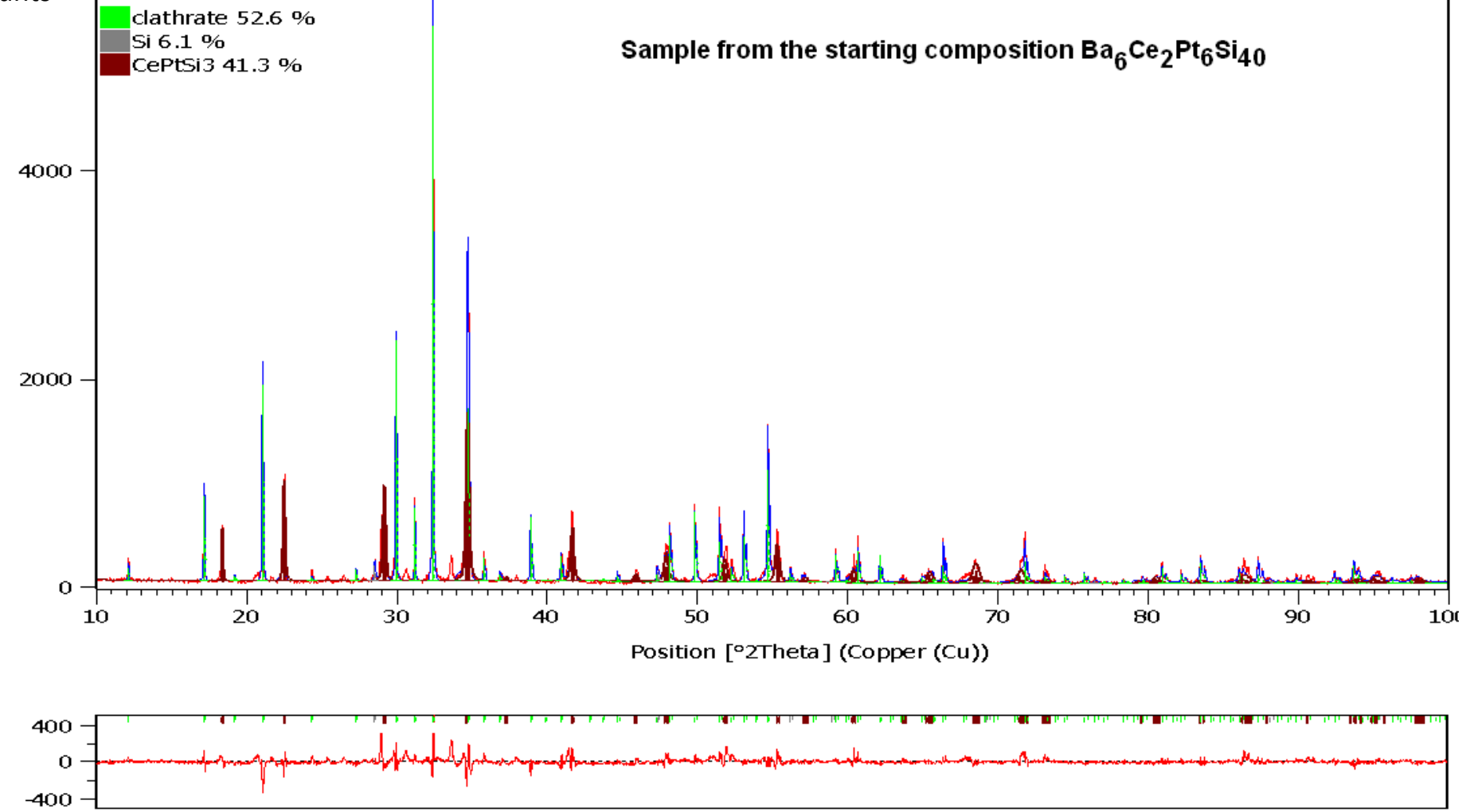

1. Si,

2. (Ce, Ba)PtSi ${ }_{3}$ (S.G. $14 m m$ ). Structure type $\mathrm{BaNiSn}_{3}$ (Ref. 21)

$$
\begin{aligned}
& a=4.3393(3) \AA \\
& c=9.6633(8) \AA,
\end{aligned}
$$

3. A small amount of a not identified phase 
$\mathrm{TM}=\mathbf{P d}$
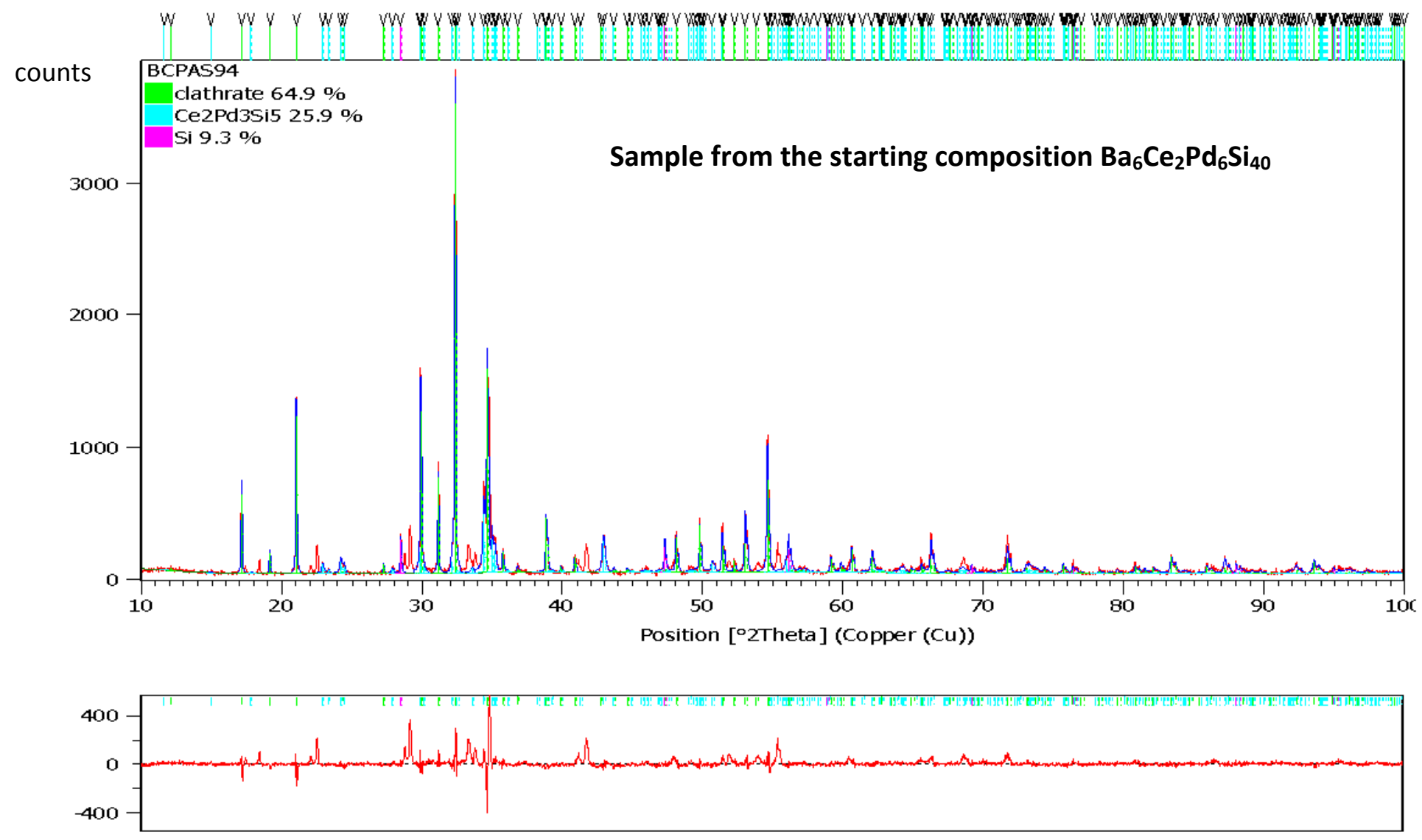

\section{$\mathrm{Ba}_{6} \mathrm{Ce}_{2} \mathrm{Pd}_{6} \mathrm{Si}_{40}$}

Clathrate phase $(\mathrm{Ba}, \mathrm{Ce})_{8.00} \mathrm{Pd}_{3.19} \mathrm{Si}_{42.81}$

$a=10.3561(2) \AA$

Secondary Phases:

1. $\mathrm{Si}$,

2. (Ce, Ba) $)_{2} \mathrm{Pd}_{3} \mathrm{Si}_{5}$ (S.G. Ibam). Structure type $\mathrm{U}_{2} \mathrm{Co}_{3} \mathrm{Si}_{5}$ (Ref. 22)

$a=9.9577(3) \AA$

$b=11.8635(4) \AA$

$c=5.9820(4) \AA$,

3. A small amount of a not identified phase 
Typical Laue pattern of a single crystal $\mathrm{Ba}_{8-x} \operatorname{La}_{x} \mathrm{Au}_{\mathrm{y}} \mathrm{Si}_{46-\mathrm{y}}$.

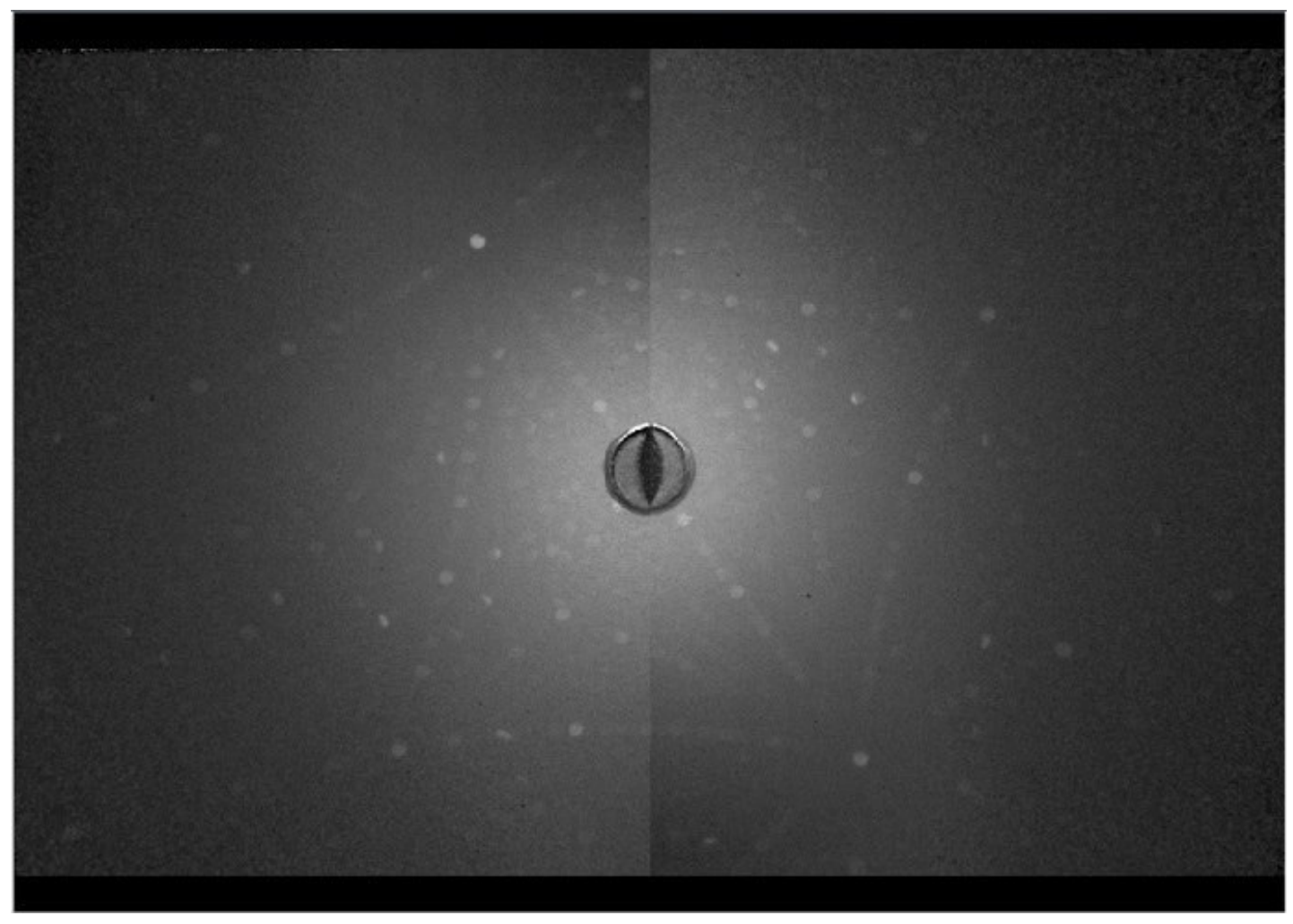

Summary guest and host local element contents for samples 1-7 (in the clathrate phase).

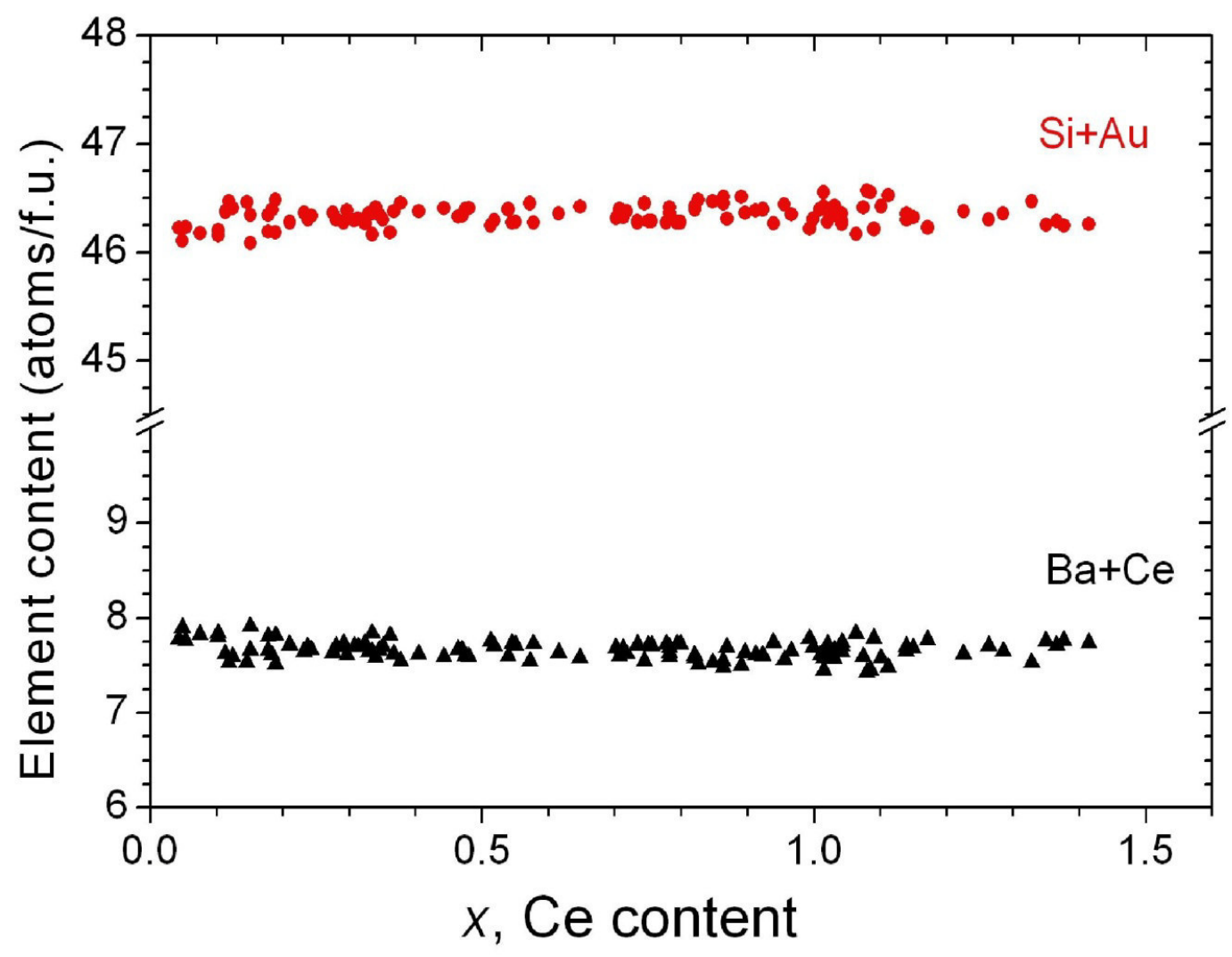


Comparison of the EDX and ICP-MS measurements of the same La-containing clathrate single crystal

\begin{tabular}{|c|c|c|}
\hline & EDX & $\begin{array}{c}\text { ICP-MS } \\
\text { (kindly provided by Yu.Grin, Max- } \\
\text { Planck-Institut für Chemische } \\
\text { Physik fester Stoffe) }\end{array}$ \\
\hline $\mathrm{Ba}$ & 6.29 & 6.183 \\
\hline $\mathrm{La}$ & 1.39 & 1.385 \\
\hline $\mathrm{Au}$ & 5.64 & 5.589 \\
\hline $\mathrm{Si}$ & 40.69 & 40.840 \\
\hline
\end{tabular}

Comparison of compositions measured by EDX and by single crystal XRD refinement carried out on the same RE-containing clathrate single crystalline samples

\begin{tabular}{|l|l|l|l|l|l|l|l|l|}
\hline & \multicolumn{2}{|c|}{ Ce } & \multicolumn{2}{c|}{ Pr } & \multicolumn{2}{c|}{ Sm } & \multicolumn{2}{c|}{ Yb } \\
\hline & EDX & scXRD & EDX & scXRD & EDX & scXRD & EDX & scXRD \\
\hline $\mathrm{Ba}$ & 6.6 & 7.3 & 6.8 & 7.4 & 7.1 & 7.8 & 7.43 & 7.86 \\
\hline $\mathrm{RE}$ & 1.1 & 0.7 & 1.0 & 0.6 & 0.4 & 0.2 & 0.36 & 0.14 \\
\hline $\mathrm{Au}$ & 5.7 & 5.9 & 5.5 & 5.7 & 4.8 & 5.4 & 5.46 & 5.32 \\
\hline $\mathrm{Si}$ & 40.6 & 40.1 & 40.7 & 40.2 & 41.7 & 40.6 & 40.76 & 40.68 \\
\hline
\end{tabular}

